\title{
THE 1957 NOVEMBER 4 EVENT
}

\author{
A. BOISCHOT \\ Observatoire de Meudon, Meudon (Seine-et-Oise), France \\ A. D. FOKKER \\ Ionosphere and Radio Astronomy Section, Netherlands Postal \\ and Telecommunications Services
}

The Hague, The Netherlands

On 1957 November 4, an unprecedented solar radio event was observed at meter wavelengths. Observations at $169 \mathrm{Mc} / \mathrm{s}$ were obtained by the Nançay Observatory (great interferometer and simple radio telescope) and by the Humain Observatory. At $200 \mathrm{Mc} / \mathrm{s}$ the event was observed by the Nera Observatory.

The phenomenon was exceptional in several respects :

(1) The very great intensity, which was maintained during a considerable time interval (about an hour). At $200 \mathrm{Mc} / \mathrm{s}$ the greatest intensity amounted to almost 1000 times the quiet sun radio emission.

(2) The very peculiar intensity variations, which were superimposed upon the enhanced flux level. Their character was altogether different from the type of variability of noise storms. The first part of the event showed very rapid intensity fluctuations (about 5 per second); during the second part great variations of intensity with a duration of a few seconds prevailed.

(3) The complete lack of concomitant phenomena. The beginning at $08^{\mathrm{h}} 40^{\mathrm{m}}$ U. T. did not coincide with the occurrence of a solar flare or with a sudden ionospheric disturbance. Nor were geomagnetic or cosmic ray effects observed. Also unusual is the fact that this large radio event was restricted to the meter wavelengths. At and beyond $545 \mathrm{Mc} / \mathrm{s}$ solar radiation was perfectly calm. Neither was a disturbance observed at a wavelength of 6 meters.

\section{EVOLUTION OF THE EVENT}

The type of radiation characteristic for the event was first observed at $08^{\mathrm{h}} 40^{\mathrm{m}}$ U. T. The radiation was only slightly enhanced until $09^{\mathrm{h}} 15^{\mathrm{m}}$, when there occurred a steep rise of intensity. For about one hour the flux level was very high (100 to 900 times the quiet sun flux level). It was subject to very large intensity variations with durations of the order of a few minutes. After $10^{\mathrm{h}} 15^{\mathrm{m}}$ the mean flux was at a lower level, some 10 to 150 times the quiet sun. The intensity variations, however, continued to be very important.

During the early stages of the event the very short periodic intensity variations showed a symmetrical pattern around the mean intensity level, 
increases and decreases occurring in equal amounts and magnitudes. They were fiercest during the period $09^{\mathrm{h}} 00^{\mathrm{m}}-09^{\mathrm{h}} 30^{\mathrm{m}}$. A fragment of a high-speed record (speed : $5 \mathrm{~mm} / \mathrm{second}$ ) obtained at $200 \mathrm{Mc} / \mathrm{s}$ is reproduced in Fig. 1. High-speed records $(5 \mathrm{~mm} / \mathrm{second})$ at $169 \mathrm{Mc} / \mathrm{s}$ showed the same characteristics. This type of intensity fluctuation is strongly reminiscent of ionospheric scintillations. After $09^{\mathrm{h}} 30^{\mathrm{m}}$ the oscillation-like intensity fluctuations occurred more intermittently. At the same time another feature gradually began to

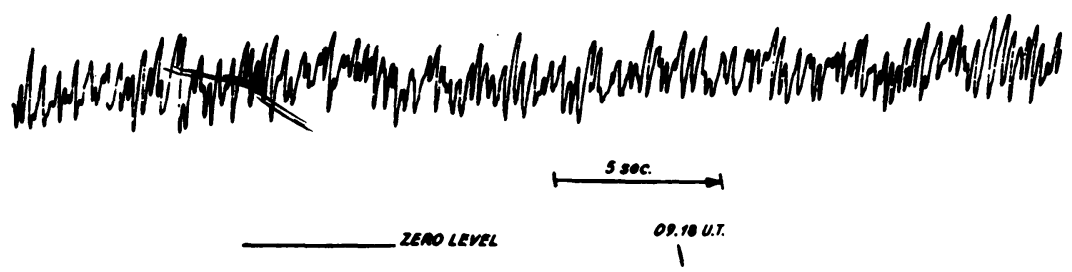

FIG. 1. High-speed record at $200 \mathrm{Mc} / \mathrm{s}$, showing extremely rapid intensity variations.

develop. During certain periods short dips of intensity prevailed above increases of intensity. They had the appearance of bursts in absorption. Sometimes there was an alternation of short periods ( $\sim 1$ minute) during which the intensity variations were preferably downward or upward. These alternations seemed to be related to a corresponding rise and fall of the mean flux level (Fig. 2).

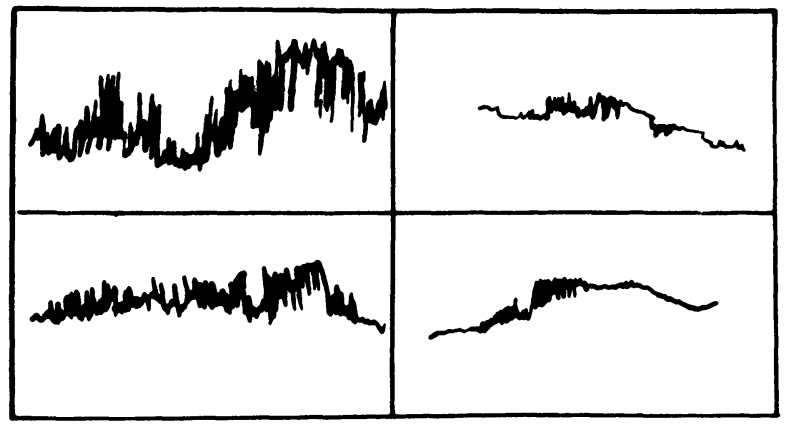

FIG. 2. 169-Mc/s record showing "bursts in absorption."

In a still more advanced stage of the phenomenon (later than about $11^{\mathrm{h}} 45^{\mathrm{m}}$ ) another type of equally peculiar intensity fluctuations of somewhat longer duration (a few seconds) was observed together with the very short periodic ones, which gradually became less important (Fig. 3). These longer-duration intensity fluctuations continued to occur intermittently up to about $15^{\mathrm{h}} 00^{\mathrm{m}}$. At about $15^{\mathrm{h}} 15^{\mathrm{m}}$ the phenomenon had come to an end.

\section{ADDITIONAL INFORMATION}

The slow over-all intensity variations at $200 \mathrm{Mc} / \mathrm{s}$ and those at $169 \mathrm{Mc} / \mathrm{s}$ (as observed by the Humain Observatory, courtesy Dr. Coutrez) resemble each 


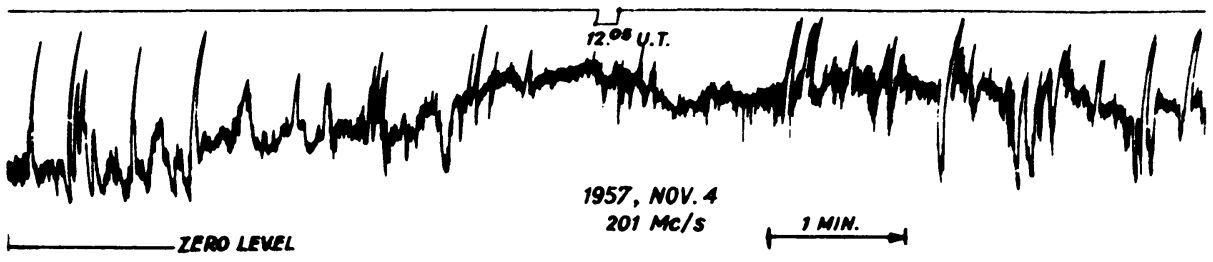

FIG. 3. $200-\mathrm{Mc} / \mathrm{s}$ record, showing peculiar intensity variations of a few seconds' duration.

other to some extent. Sometimes the two records seem to agree in a particular rise and fall of intensity. In those cases where such an identification seems possible, there often was a difference in time of one or two minutes between corresponding features. This was particularly so between $09^{\mathrm{h}} 45^{\mathrm{m}}$ and $11^{\mathrm{h}} 00^{\mathrm{m}}$, when the $169-\mathrm{Mc} / \mathrm{s}$ features seem to precede the $200-\mathrm{Mc} / \mathrm{s}$ ones. Because of the many differences in detail, however, it is very hard to judge what significance should be attached to the general correspondence and to the systematic time difference.

Whether there was a correlation between the very-short-period intensity fluctuations at the two frequencies could not be ascertained, because the Nançay and Nera high-speed records covered different parts of the event.

Observations with a radio spectrograph, obtained from $14^{\mathrm{h}} 20^{\mathrm{m}}$ on, showed a very unusual spectrum (F. T. Haddock, private information). There were bursts, lasting up to about one minute, which were subject to very irregular spectral variations. Their bandwidth was extremely small, of the order of $1 \mathrm{Mc} / \mathrm{s}$. That the bandwidth must have been very small also appeared from simultaneous measurements with the great Nançay interferometer at two frequencies which differed by $1.8 \mathrm{Mc} / \mathrm{s}$.

The radiation was uniformly 100 per cent left-handed polarized during the whole evolution of the event at $200 \mathrm{Mc} / \mathrm{s}$.

Determining the source position at $169 \mathrm{Mc} / \mathrm{s}$ with the great interferometer is rather difficult because of the continuous changes of intensity. The source seems to have been situated a few minutes of arc to the west of the northsouth diameter of the solar disk (Fig. 4). At this position a small flare was observed at $09^{\mathrm{b}} 37^{\mathrm{m}}$ which lasted until $10^{\mathrm{h}} 04^{\mathrm{m}}$. The occurrence of this flare may very well have been accidental, having no relation to the great radio event. A two-element interferometer measurement at Nera yielded a position approximately 3 minutes of arc east of the north-south diameter at $255 \mathrm{Mc} / \mathrm{s}$. The position remained stationary during two hours centered around local noon $\left(11^{\mathrm{h}} 23^{\mathrm{m}} \mathrm{U} . \mathrm{T}\right)$. The passages through zero of the two-element interferometer record (time constant 1 second) succeeded each other on very regular time intervals. Consequently, the momentary position of the effective center of the emissive region certainly cannot have fluctuated by more than \pm 0.8 minutes of arc around the mean position.

The character of the record obtained with the great interferometer suggests that small patches of considerable brightness were superposed upon the 


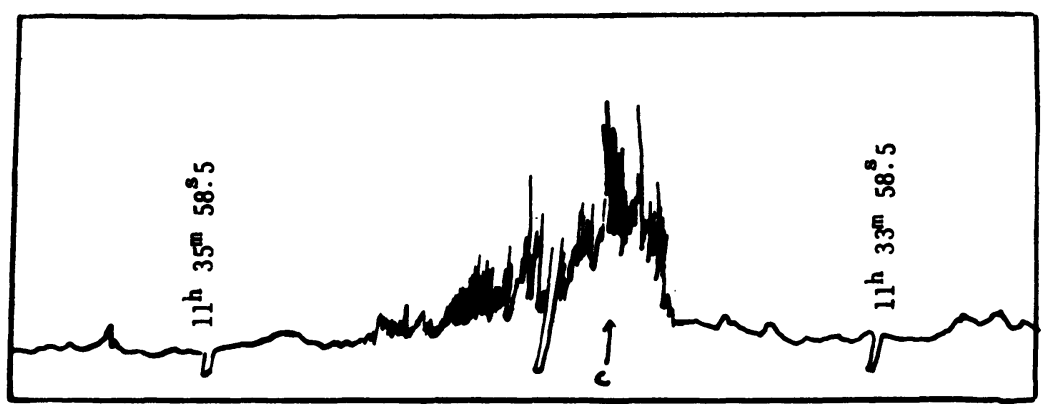

Fig. 4. Record obtained with the great Nançay interferometer at $169 \mathrm{Mc} / \mathrm{s}$ during meridian passage of the sun.

emissive region. The extent of this region seems to have been considerable, about 9 minutes of arc.

\section{DISCUSSION}

A very special condition on the sun must have reigned under which this very unusual radio event could develop. It does not fit into any of the types of outbursts recognized up to now. Because no similar event has ever been observed in the past and owing to lack of correlation with optical or terrestrial phenomena, it is very difficult to indicate what kind of process might have produced this special type of emission. The process evidently underwent an evolutionary development, because the characteristics of the event showed a systematic and gradual change. Something similar to the bursts in absorption occurred once during about 0.5 minutes in the first part of an outburst of 4 minutes duration. This rather special feature is confirmed by Cornell University records.

Particularly difficult is the interpretation of the very-short-period oscillations and fading-like intensity fluctuations. One wonders whether sources of a very small angular dimension could be liable to scintillation effects produced by small ionospheric irregularities. The short-period fluctuations were definitely too fast to ascribe them to the scintillation that is commonly displayed by radio sources. According to routine scintillation observations at Jodrell Bank and at Cambridge the scintillation conditions were very quiet on November 4. However, some kind of scintillation mechanism might have been at work in the solar corona.

It is not entirely out of the question that certain intensity fluctuations that occurred later than $11^{\mathrm{h}} 00^{\mathrm{m}}$ were due to scintillation, because the correlation between the 169-Mc/s Humain and Nançay records was definitely less good than during the first part of the radio event. This might also be the consequence of a slight difference in frequency of the receivers, combined with the very small bandwidth of the features. However, it is surprising that there were even notable differences between the detailed intensity fluctuations observed at Nancay with the great interferometer and with a simple radio 
telescope, although the two receivers were tuned at the same frequency, with identical bandwidths!

We dare not estimate how long we shall have to wait for the next event of this type to occur. Perhaps still other types of radio emission will surprise solar radio observers on most unexpected occasions!

We are indebted to numerous observatories for information on their observations on November 4. We specially wish to thank Dr. R. A. J. Coutrez of the Humain Observatory, Uccle, and Dr. F. T. Haddock of the University of Michigan Observatory for making their observations available to us.

\section{Discussion}

Kaiser: There occurred on the night of November $6 / 7$ a bright aurora visible in northern England.

Fokker: The auroral display of 1957 November 6 probably is to be ascribed to a solar flare of importance 2 which occurred on November 5 at $12^{\mathrm{b}} 05^{\mathrm{m}}$ and which produced strong radio responses at 200,545 , and $2980 \mathrm{Mc} / \mathrm{s}$ ("radio importance" 3).

Roberts: I would like to make two comments. First, what we believe to be ionospheric scintillations are sometimes observed in type I radiations. These appear as both positive and negative variations of intensity and have durations of about two minutes. They resemble very closely the spectra of the scintillations of the radio source in Cygnus (Wild and Roberts, J. Atmos. Terr. Phys. 8, 55, 1956). Second, some type I storms are made up of bursts of very short duration (less than one second) and broad bandwidths (tens of $\mathrm{Mc} / \mathrm{s}$ ). These bursts may have either positive or negative drifts at rates similar to type III bursts.

Haddock: The dynamic spectra (100 to $580 \mathrm{Mc} / \mathrm{s}$ ) of the latter part of this event showed unique narrow-band "wavy lines" drifting first to higher and then to lower frequencies, some "lines" in emission and some in absorption. No observations could be made of the beginning of the event at our longitude. No other records during eleven months of recording displayed similar spectra. 